\section{Social Attention Promotes Partner Intimacy}

\author{
Yohsuke Ohtsubo ${ }^{1, *}$, Shiori Tamada ${ }^{2}$ \\ ${ }^{1}$ Department of Psychology, Graduate School of Humanities, Kobe \\ University, Kobe 657-8501, Japan. \\ ${ }^{2}$ Department of Psychology, Faculty of Letters, Kobe University, Kobe 657- \\ 8501, Japan. \\ "Author for correspondence (yohtsubo@lit.kobe-u.ac.jp)
}

Social animals develop intimate bonds with their social partners. However, bond formation entails the risk of being exploited by partners. Previous studies have shown that people monitor partner attention to themselves to assess commitment to the relationship. Accordingly, a partner's social attention promotes the receiver's intimacy with the partner. This study expanded previous finding by manipulating partner attention in a naturalistic manner. In particular, naïve participants were assigned to one of two roles (i.e., signal Sender and Receiver) in the laboratory. Receiver first wrote a self-instruction essay, and Sender read it under either of two instructions: to pay close attention to the content of the essay (the high attention condition) or to some peripheral aspects of the essay (the low attention condition). After reading the essay, Sender's memory of the essay was assessed. Naturally, Sender recalled it more accurately in the high attention condition. Knowing Sender's accuracy, Receiver reported a sense of intimacy toward Sender. Receiver's intimacy was higher in the high attention condition than in the low attention condition. Therefore, this study confirmed that paying attention to a partner, which translates to accurate understanding of the partner, promotes the intimacy in the partner.

\section{Keywords}

social attention, signal, intimacy

\section{Introduction}

Humans form intimate bonds not only with kin group members, but also with non-kin others. However, forming interpersonal bonds (especially with unrelated others) is associated with a difficult problem, the so-called commitment problem (Frank, 1988; Nesse, 2001). Forming committed relationships is difficult because of its apparent irrationality: Person A committing him- or herself to a particular partner (Person B) implies that A abandons more attractive partners whom he or she will encounter in the future. However, without commitment, it would be impossible for A and B to form close relationships. Moreover, the apparent irrationality causes another problem: Even when A somehow manages to commit him- or herself to the current relationship partner (B), B may doubt A's commitment. Accordingly, to fully solve the commitment problem, A has to be able to credibly communicate his or her commitment to B. For this reason, the costly signaling theory is relevant to the commitment problem (e.g., Frank, 1988; Zahavi, 1977): If $A$ values $B$ as a relationship partner, A can signal the valuation by incurring otherwise intolerable costs, such as giving expensive flowers to B (Sozou \& Seymour, 2005). Alternatively, A might produce small signals constantly (Hruschka, 2010; Yamaguchi, Smith, \& Ohtsuob, 2015). The small signals, if produced constantly, can serve as a costly signal because producing constant signals requires time and attention to the partner, both of which are limited resources.

Recently, Ohtsubo et al. (2014) revealed that A's action of paying attention to B promotes B's intimacy with A. In a series of vignette studies, Ohtsubo et al. manipulated the presence of attention involved in an imaginary partner's pro-relationship behaviors. Participants who read the same pro-relationship behaviors indicated a greater sense of intimacy with the imaginary partner when attention was involved in the behaviors. In a second set of experimental studies, Ohtsubo et al. had participants engage in a collaborative task with a partner (in fact, a computer program). The partner's attention was manipulated by a signal placed on a computer display. The signal indicated whether the putative partner was monitoring the participants' task. Participants who believed that their partner had frequently monitored them indicated greater intimacy with the partner than those who believed that their partner had not monitored them.

One problem pertinent to Ohtsubo et al.'s (2014) experimental studies is the artificial nature of the partner's attention (i.e., the blue/red signal on the computer display). Therefore, the present study attempted to conceptually replicate Ohtsubo et al.'s result employing a more naturalistic manipulation of partner attention. In the present study, we divided participants into two roles (i.e., Sender and Receiver of a commitment signal) and directly manipulated Sender's attention to Receiver. In particular, Receiver first wrote a self-introduction essay. Sender read the essay under one of two instructions: To pay close attention to the content of the essay (high attention condition) or to some peripheral aspects of the essay (low attention condition). Sender was then asked to recall the contents of the essay. It is naturally expected that Sender's recall would be more accurate in the high attention condition. Furthermore, if Receiver in fact uses the accuracy as a cue to infer Sender's attention, it is expected that Sender's accuracy predicts Receiver's intimacy with Sender. This prediction was tested by assigning both Sender and Receiver roles to naïve participants. 


\section{Method}

(a) Participants and design

Participants were 60 undergraduate students at Kobe University (31 females and 29 males, mean age $\pm S D=$ $19.71 \pm 1.41$ years). The experiment consisted of six sessions, each of which involved seven to 14 participants. Two of the participants in each session were chosen as Senders in a semi-random manner. In each session, one of the two Senders was assigned to the high attention condition and the other to the low attention condition. The remaining five to 12 participants in each session were assigned to the role of Receiver. They were divided into the two attention conditions. Accordingly, Each Sender was paired with two to six Receivers. This procedure yielded 12 Senders ( 6 each for the high and low attention conditions) and 48 Receivers. Although participants first met in the same room, they were unable to identify their partner(s) because there were more than one Senders and Receivers in each session.

\section{(b) Procedure}

After participants arrived, the experimenter explained the nature of the experiment, and obtained signed informed consent forms from participants. The experimenter asked whether there were mutual friends in the session. Mutual friends were assigned to the role of Sender to ensure anonymity (if mutual friends were paired as Sender and Receiver in the experiment, they might be able to identify their experimental partner). When there were no mutual friends, two participants were assigned to the role of Sender by a random lottery.

Receivers first wrote a self-introduction essay. They were explicitly required to include 15 pieces of information in the essay (e.g., hobby, favorite sports, see the Electronic Supplementary Materials for more details). Receivers' essays were then given to their paired partner (i.e., Sender). In the high attention condition, Senders were instructed to pay close attention to the information described in the essay. In the low attention condition, Senders were asked to search for and circle certain letters (i.e., “は” (ha or wa) and “た" (ta) including their voiced-sound forms, "ba," "pa," and "da"). After 90 seconds elapsed for each essay, Senders were asked to recall and write the 15 pieces of information on the answer sheet. Each Receiver was given the answer sheet filled out by his or her Sender and graded it without knowing the instructions given to Senders. Therefore, Receivers became aware of their Sender's memory accuracy, and were likely to attribute Sender accuracy to Sender attention to them. Receivers then filled out the questionnaire containing the intimacy items (see the following "dependent variables" section). Finally, each Receiver engaged in a modified version of the dictator game with his or her Sender, whereby Receiver unilaterally allocated a fixed amount of money between him- or herself and his or her Sender. However, the dictator game did not yield any meaningful difference. Therefore, we do not report the result of dictator game in the main text. Interested readers can find the details of this game in the electronic supplementary materials. Participants were fully debriefed after the dictator game and received their rewards in accordance with the result of the dictator game.

\section{(c) Dependent variables}

The primary dependent variable was Receivers' intimacy with Sender. The questionnaire included four intimacy items corresponding to the three components of Reis and Shaver's (1988) definition of intimacy: understanding (If you became friends with the partner, how well do you think this person would understand you?), validation (If you became friends with the partner, how much do you think this person would accept you?), and caring (How much did the partner care for you?). There was an additional reversed-coded caring item (How much do you agree that this partner had little concern for you?). The responses to these four items, measured on a 7-point scale, were aggregated as the intimacy score (Cronbach's alpha = $.74)$.

In addition to the intimacy items, the questionnaire included items assessing the recipients' interests in developing a friendship with their Sender: "How much are you interested in being friends with the partner?" and "How much do you agree that the partner and you will be good friends?" The responses to these two items were aggregated as the interest in friendship score (the interitem correlation $=.50, d f=46, p<.001$ ). In addition, there were two items assessing Receivers' positive evaluation of their Senders: "My partner [i.e., Sender] is a nice person" and "My partner is a helpful person." The responses to these two items were aggregated as the favorable impression score (the inter-item correlation $=.77, d f=46, p$ $<.001)$. It was expected that intimacy would foster interest in friendship with Sender and a favorable impression of Sender.

An additional dependent variable of this experiment was Sender accuracy, which could range from 0 to 15 . We tested whether distracted attention would in fact result in low accuracy in the understanding of the essay.

\section{Results}

\section{(a) Sender accuracy}

We first confirmed that Sender accuracy was in fact reduced by distracted attention. As shown in Figure 1, the distributions of Sender accuracy in the high and low attention conditions did not overlap. Because each

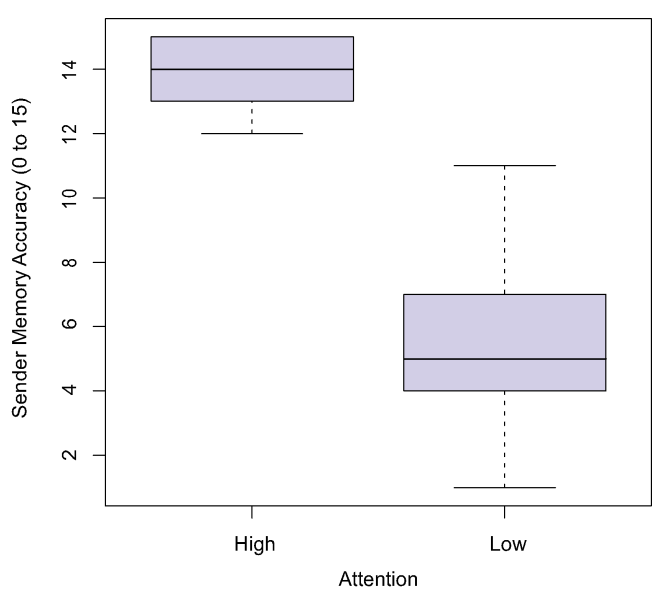

Figure 1. Distributions of the Sender accuracy scores (theoretical range $=0$ to 15 ) in the high and low attention conditions. 
Sender was paired with multiple Receivers, the accuracy scores were averaged within each Sender. The mean accuracy score $\pm S D$ was $14.09 \pm 0.72$ and $5.11 \pm 2.18$ in the high and low accuracy conditions, respectively, $t(6.07)=$ $9.58, p<.001$ by Welch's test. The attention instructions successfully translated to the accuracy score.

\section{(b) Hypothesis testing}

Three Receiver variables (i.e., intimacy, interest in friendship, favorable impression) are highly correlated with each other (see the correlation matrix, Table S1, in the Electronic Supplementary Materials). Therefore, instead of testing the attention effect on each variable, we tested the effect of attention on the set of the three variables by a 2 (condition) $\times 2$ (sex) multivariate analysis of variance (MANOVA). The effect of attention was significant, $F(3$, $42)=8.47, p<.001$, while other effects were not significant. As shown in Figure 2, the mean intimacy, interest in friendship, and favorable impression scores were higher in the high attention condition $(5.15 \pm 0.69$ vs. $4.07 \pm 0.80$ for intimacy; $4.44 \pm 1.15$ vs. $3.81 \pm 0.96$ for interest in friendship; $4.90 \pm 1.14$ vs. $3.77 \pm 0.85$ for favorable impression). A series of follow-up t-tests with the Bonferroni correction (the significance level was set at .0167) revealed the significant attention effect on intimacy and favorable impression, $t(46)$ $=5.00, p<.001$, Cohen's $d=1.45$, and $t(46)=3.88, p<$ .001 , Cohen's $d=1.12$, respectively. However, the effect of attention on interest in friendship was not significant, $t(46)$ $=2.04, p=.047$, Cohen's $d=0.59$. In sum, the primary hypothesis (i.e., partner attention promotes intimacy) was supported, and one of the auxiliary hypotheses (i.e., partner attention promotes a favorable impression of the partner) was supported.

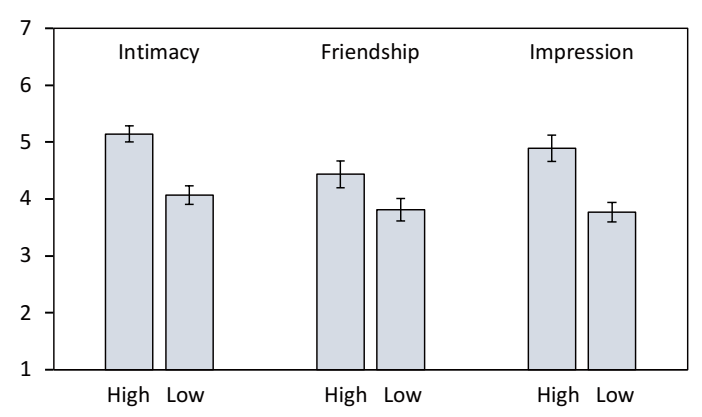

Figure 2. Mean intimacy with Sender, interest in friendship with Sender, and favorable impression of Sender as a function of the attention condition (high vs. low). All these are measured with a seven-point scale $(1=$ "not at all" to 7 $=$ "very much"). Error bars indicate the standard errors of the means.

\section{(c) Mediation analysis}

We then tested whether the effect of attention on a favorable impression would be mediated by intimacy. A mediation analysis using the bootstrapping method revealed that the attention-favorable impression relationship was fully mediated by intimacy (the 95\% CI of the indirect effect was .16 to .34: Figure 3).

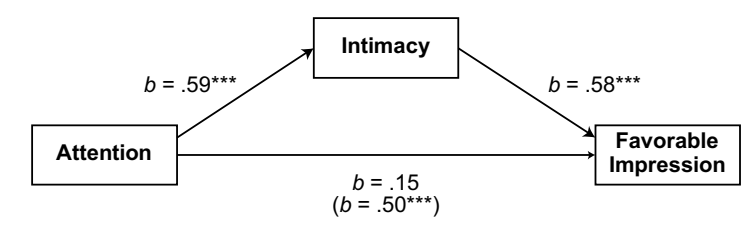

Figure 3. Intimacy mediates the relationship between Sender attention and Receiver favorable impression. The path coefficients are standardized. "***” designates " $p<$ .001."

\section{Discussion}

This study expanded Ohtsubo et al.'s (2014) finding that a partner's attention fosters intimacy with the partner by naturalistically manipulating the partner's attention. These results seem to suggest that people are closely tracking partner attention directed toward them, and adjust their intimacy with the partner accordingly. Moreover, how the partner attention is expressed does not matter: As far as it is recognized as attention, the intimacy-fostering effect persists. Although Ohtsubo et al. (2014) reported that intimacy was positively correlated with participants' willingness to engage in generous exchanges with their partners, the dictator game included in this study did not yield any meaningful finding (reported in the Electronic Supplementary Materials). More sensitive behavioral measures are needed in future research.

In addition, the external validity of the finding needs to be investigated. For example, it is necessary to examine whether mutual friends or romantic partners in fact pay more attention to their intimate partners than to nonintimate others. As the signaling theory assumes the co-evolution of signal senders and receivers, it is also necessary to examine whether friends or romantic partners accurately track their partner attention (see Yamaguchi et al., 2015, for suggestive evidence). Finally, the correlation between A's attention and B's intimacy in real relationships must be assessed.

\section{Acknowledgements}

We are grateful to Asuka Narita, Yui Tanaka, and Shoko Tsuda for their assistance in data collection. This research was generously supported by the Japan Society for the Promotion of Science to Yohsuke Ohtsubo (JSPS KAKENHI Grant No. 26590132).

\section{References}

Frank, R. H. (1988). Passions within reason: The strategic role of the emotions. New York: Norton.

Hruschka, D. J. (2010). Friendship: Development, ecology, and evolution of a relationship. Berkeley, CA: University of California Press.

Nesse, R. M. (Ed.). (2001). Evolution and the capacity for commitment. New York: Russell Sage Foundation.

Ohtsubo, Y., Matsumura, A., Noda, C., Sawa, E., Yagi, A., \& Yamaguchi, M. (2014). It's the attention that counts: Interpersonal attention fosters intimacy and social exchange. Evolution and Human Behavior, 35, 237244. (doi: 10.1016/j.evolhumbehav.2014.02.004) 
Reis, H. T., \& Shaver, P. (1988). Intimacy as an interpersonal process. In S. W. Duck (Ed.), Handbook of personal relationships: Theory, research and interventions (pp. 367-389). Chichester, England: Wiley.

Sozou, P. D., \& Seymour, R. M. (2005). Costly but worthless gifts facilitate courtship. Proceedings of the Royal Society B: Biological Sciences, 272, 1877-1884. (doi: 10.1098/rspb.2005.3152)

Yamaguchi, M., Smith, A., \& Ohtsubo, Y. (2015). Commitment signals in friendship and romantic relationships. Evolution and Human Behavior, 36, 467-474. (doi: 10.1016/j.evolhumbehav.2015.05.002)

Zahavi, A. (1977). The testing of a bond. Animal Behaviour, 25, 246-247. (doi: 10.1016/00033472(77)90089-6) 